\title{
Alternative lengthening of telomeres in molecular subgroups of paediatric high-grade glioma
}

\author{
Simone Minasi ${ }^{1,2}$ (D) Caterina Baldi ${ }^{2}$ (D) $\cdot$ Francesca Gianno ${ }^{1,3}$ (D) $\cdot$ Manila Antonelli ${ }^{1}$ (D) Anna Maria Buccoliero ${ }^{4}$ (D) \\ Torsten Pietsch $^{5}$ (1D $\cdot$ Maura Massimino ${ }^{6}$ (D) . Francesca Romana Buttarelli ${ }^{1,2}$ (D)
}

Received: 7 April 2020 / Accepted: 16 October 2020 / Published online: 31 October 2020

(C) The Author(s) 2020

\begin{abstract}
Purpose The maintenance of telomere length prevents cancer cell senescence and occurs via two mutually exclusive mechanisms: (a) reactivation of telomerase expression and (b) activation of alternative lengthening of telomeres (ALT). ALT is frequently related to alterations on ATRX, a chromatin-remodelling protein. Recent data have identified different molecular subgroups of paediatric high-grade glioma (pHGG) with mutations of H3F3A, TERTp and ATRX; however, differences in telomere length among these molecular subgroups were not thoroughly examined.

Methods We investigated which genetic alterations trigger the ALT mechanism in 52 IDH-wildtype, 1p/19q-wildtype pHGG. Samples were analysed for telomere length using Tel-FISH. ATRX nuclear loss of expression was assessed by IHC, $H 3 F 3 A$ and TERTp mutations by DNA sequencing, and TERTp methylation by MS-PCR.

Results Mutant H3.3 was found in 21 cases (40.3\%): $19.2 \%$ with K27M mutation and $21.1 \%$ with G34R mutation. All H3.3G34R-mutated cases showed the ALT phenotype (100\%); on the opposite, only $40 \%$ of the H3.3K27M-mutated showed ALT activation. ATRX nuclear loss was seen in 16 cases (30.7\%), associated sometimes with the G34R mutation, and never with the K27M mutation. ATRX nuclear loss was always related to telomere elongation. TERTp C250T mutations were rare (5.4\%) and were not associated with high intensity Tel-FISH signals, as TERTp hyper-methylation detected in $21 \%$ of the cases. H3.3/ ATRX/TERTp-wildtype $\mathrm{pHGG}$ revealed all basal levels of telomere length.

Conclusion Our results show a strong association between H3.3 mutations and ALT, and highlight the different telomeric profiles in histone-defined subgroups: H3.3-G34R mutants always trigger ALT to maintain telomere length, irrespective of ATRX status, whereas only some H3.3-K27M tumours activate ALT. These findings suggest that acquiring the gly34 mutation on H3.3 might suffice to trigger the ALT mechanism.
\end{abstract}

Keywords Telomeres $\cdot$ Alternative lengthening of telomeres $\cdot$ H3.3 $\cdot$ Paediatric high-grade gliomas $\cdot$ pHGG

Simone Minasi and Caterina Baldi contributed equally to this work.

Supplementary Information The online version of this article (https:// doi.org/10.1007/s00381-020-04933-8) contains supplementary material, which is available to authorized users.

Francesca Romana Buttarelli

francesca.buttarelli@uniroma1.it

1 Department of Radiological, Oncological and Anatomo-Pathological Sciences, Sapienza University of Rome, Rome, Italy

2 Department of Human Neurosciences, Sapienza University of Rome, Rome, Italy

3 Department of Molecular Medicine, Sapienza University of Rome, Rome, Italy
4 Pathology Unit, Meyer Children's University Hospital, I-50139 Florence, Italy

5 Institute of Neuropathology, DGNN Brain Tumour Reference Centre, University of Bonn Medical Centre, Bonn, Germany

6 Paediatric Unit, Fondazione IRCCS Istituto Nazionale dei Tumori, Milano, Italy 


\section{Introduction}

Gliomas account for $\sim 11 \%$ of all central nervous system (CNS) tumours in children aged 0-14 years. Paediatric highgrade glioma ( $\mathrm{pHGG)}$ is one of the most common causes of cancer-related death under the age of 19 [42]. Despite histological similarities between $\mathrm{pHGG}$ and adult malignant gliomas, the former are more widely distributed within the CNS, with approximately $50 \%$ of cases occurring in midline locations [24].

The recent extensive use of high-throughput molecular, genetic and epigenetic profiling has considerably increased our knowledge of the cellular origin, pathogenesis and biological features of pHGG. This has helped to classify these neoplasms by their molecular/genetic features, which correlate with age of onset, anatomical location and prognosis $[5,11$, $35,40,45,51]$.

The maintenance of telomere length is a major molecular factor in human cancer development. Unlimited replication and immortalization are key features of neoplastic cells, needed to escape replicative senescence due to telomere shortening $[41,47]$. Telomere length can be maintained via two different mechanisms: (i) reactivation of telomerase reverse transcriptase (TERT) via promoter mutations or methylation; and (ii) a telomerase-independent mechanism known as alternative lengthening of telomeres (ALT), which relies on the homologous recombination of telomeric regions $[1,2,5,19,20,26$, 38 ], and results in a heterogeneous length and sequence composition $[19,20,26]$.

Compared with adult HGG, pHGG differ in the frequency of isocitrate dehydrogenase $(I D H 1 / 2)$ mutations $(<6 \%$, mostly in adolescents) $[8,35,57]$, and telomerase reverse transcriptase promoter (TERTp) mutations (5 vs. $30-50 \%$ in adults) $[11,23,26,30,35]$. Hotspot mutations in histone H3.3 encoding gene (H3F3A) have also been found in $>50 \%$ of pHGG $[35,48]$, whereas they are rare in adult gliomas. With respect to this latter biomarker, the H3.3-K27M hotspot mutation is a characteristic of the "diffuse midline glioma (DMG) H3-K27M-mutant" [35, 51]. The clinico-pathological and biological significance of H3.3-G34R/V has yet to be fully elucidated [55], but this mutation will probably identify a separate biological entity in upcoming classifications.

The most common alterations related to telomerase upregulation are hotspot-activating mutations on TERTp $\mathrm{C} 228 \mathrm{~T}$ and C250T [5, 12, 25, 26, 30, 39]. Hyper-methylation of TERTp on the CpG-rich region - 600 bp upstream from the transcription start site (UTSS), which has been identified in paediatric brain tumours, may increase telomerase expression $[4,30]$. TERT amplification and rearrangement are virtually absent in $\mathrm{pHGG}$ [2].

Tumours that do not activate telomerase may trigger the ALT phenotype. ALT is a recombination-based mechanism, where one telomere uses other chromosomal or extra- chromosomal telomeric DNA sequences as a template for telomere elongation $[14,20,36,38]$. There are several hallmarks of the telomere length maintenance via ALT, including the following: (i) a long and heterogeneous telomere length $[14,19,20,54]$; (ii) the presence of ALT-associated promyelocytic leukaemia (PML) nuclear bodies (APBs) [28, 54]; and (iii) the generation of high levels of $\mathrm{C}$-rich circular telomeric DNA repeats (C-circles) [6, 22].

In pHGG, ALT is frequently activated by alterations of $\alpha$ thalassaemia/mental retardation syndrome X-linked (ATRX) gene or, less frequently, in the $H 3 F 3 A$ gene $[12,13,18-20$, $35,46]$. Loss of ATRX has been associated directly with extensive genome rearrangement, defective double-strand break repair and telomere elongation [3, 9, 33, 46], representing a molecular surrogate for the ALT phenotype [3, 25]. In adult HGG, inactivating mutations in ATRX and activating mutations in the TERTp are mutually exclusive, providing genetic evidence of ATRX loss contributing to telomere maintenance via the ALT phenotype in this cancer subtype [25].

A few studies have explored how TERTp, ATRX, H3.3G34R/V and H3.3-K27M mutations are linked to activation of either of the two telomere maintenance mechanisms [12, 19, $46,48,49]$, but an assessment of telomere length in each molecular subgroup of pHGG is still lacking.

Hence, our present investigation study the relationships between ALT and molecular alteration in pHGG. We hypothesised that histone-defined pHGG subgroups could differ in terms of telomeric profile and ALT activation. To this end, we screened a cohort of IDH-wildtype, 1p/19qwildtype pHGG, including H3.3-mutated, ATRX-lacking, TERTp-mutated and H3.3/ATRX/TERTp-wildtype cases for presence of the ALT phenotype. To do so, we used telomere-specific quantitative FISH on formal-fixed and paraffin-embedded (FFPE) samples, identifying any ultrabright Tel-FISH signals $[19,20,27,37]$. Since IDH mutations have been found in 35\% of HGG patients over 14 years old

\section{Materials and methods}

\section{Study cohort and DNA extraction}

The study was carried out on FFPE tissue specimens from pHGG collected at the national reference centre for paediatric brain tumours at "Sapienza Università di Roma".

Tumours were classified by two neuropathologists (FG, MA) according to the World Health Organisation (WHO) classification of CNS tumours (2016), using standard histological and immunohistochemical methods (IHC) [32, 51]. Standard markers including GFAP, Ki67, IDH1-R132H and p53 were examined using IHC. As mentioned previously, all cases were negative for IDH1-R132H, as expected $[8,35,43]$. 
All FFPE tumours containing at least $70 \%$ of neoplastic cells were selected for DNA extraction with QIAamp DNA Mini Tissue Kit (Qiagen) according to the manufacturer's instructions. DNA sequencing of $I D H 1 / 2$ genes, on exon 4 , was done to rule out any point mutations in specimens from patients over 12 years old. [43]. FISH 1p-19q co-deletion was set to exclude oligodendroglial tumours (see Fig. 1 in the Supplementary material).

Informed consent was obtained from all participants or their parents before their inclusion in the study.

\section{ATRX nuclear loss, mutant H3-K27M and -G34R/V IHC}

IHC analyses were performed on 3- $\mu$ m sections of FFPE samples, using an automated immunohistochemical stainer (Leica Bond III). At least 1000 tumour cells were analysed, adopting the following scores: no expression $=0$; weak expression $=1+$; moderate expression $=2+$; strong expression $=3+$.

Loss of ATRX nuclear expression was assessed by IHC because the presence of inactivating mutations on the ATRX gene results in loss of protein nuclear expression. As previously reported, loss of ATRX nuclear immunopositivity is widely considered a surrogate for $A T R X$-inactivating mutation status [9, 13, 25, 48]. ATRX (1:1000, NBP1-83077, Novus Biologicals) was scored as positive when neoplastic cells showed a staining intensity $>1$, and cases with $\leq 15 \%$ immunopositive tumour nuclei were assumed to harbour ATRX-inactivating mutations. Endothelial cells were used for internal positive control purposes.

Selected samples $(n=18)$ were analysed using IHC with specific antibodies against H3.3 mutations. Commercially available antibodies were used to identify K27M $(0.01 \mu \mathrm{g} /$ mL, RM192, RevMAb Biosciences), G34R (1:400, RM240, RevMAb Biosciences) and G34V (1:4000, RM307, RevMAb Biosciences) mutant proteins [53].

Staining for H3.3K27M, -G34R and -G34V were scored as positive when $>15 \%$ of neoplastic cells showed nuclear immunoreactivity, associated with the negativity of internal control (endothelial cell nuclei) [53]. H3.3 status was confirmed with $H 3 F 3 A$ sequencing on all 18 samples.

\section{H3F3A and TERTp hotspot mutation pyrosequencing}

All samples were analysed using pyrosequencing to detect H3F3A; TERTp mutations were analysed via pyrosequencing for those cases where DNA was available (37/52). PCR primers were designed to amplify the TERTp region containing the $\mathrm{C} 228 \mathrm{~T}$ and $\mathrm{C} 250 \mathrm{~T}$ hotspots, corresponding to positions 124 and 146 bp upstream from the ATG site. Primers for the $H 3 F 3 A$ mutational analysis were designed to amplify the hotspot codons on exon 2, corresponding to amino acids K27 and G34.
Pyrosequencing analysis was performed immobilising single-stranded DNA templates on streptavidin-coated sepharose high-performance beads (GE Health Care) using the PSQ Vacuum Prep Tool and Vacuum Prep Worktable. Sequencing was done using PyroGold reagents on the PyroMark Q24 instrument (Biotage), according to the manufacturer's instructions. Negative controls were used to detect background signals. The pyrograms were analysed with the Pyro Mark Q24 software (Biotage) to ascertain the proportion of mutant vs. wild-type alleles according to the percentage relative peak height, as explained elsewhere [18]. All primers, PCR and sequencing conditions were as described in a previous study [37].

\section{TERTp methylation status}

UTSS methylation status on TERTp was analysed using methylation-specific PCR (see Fig. 2 in the Supplementary materials). A semi-quantitative methylation-specific polymerase chain reaction (MS-PCR) was used to establish the methylation status of five $\mathrm{CpG}$ sites $-600 \mathrm{bp}$ upstream from the TERT transcription start site (UTSS region). Bisulphite modification of DNA was conducted using the EZ DNA Methylation Kit (ZYMO Research), according to the manufacturer's instructions. The five $\mathrm{CpG}$ sites were targeted using specific primers to amplify bisulphite-modified DNA. Two pairs of forward primers and one reverse primer, specific for methylated and unmethylated alleles, respectively (Supplementary Table 1), were used. Details of the PCR, cycling condition and analysis with ImageJ Software $(\mathrm{NIH})$ are provided in a previous work [37].

\section{Telomere-specific fluorescence in situ hybridization and image analysis}

Telomere length was assessed on 5- $\mu \mathrm{m}$ FFPE sections using telomere-specific fluorescence in situ hybridization (TelFISH). Telomere length was analysed with FITC-PNA probes (K532511, Dako) complementary to the telomeric-repeated sequences, according to the manufacturer's instructions. The PNA probes do not recognise sub-telomeric sequences, enabling a precise measurement of telomere length (Dako). FISH sections were examined with an AxioImager M1 microscope (Carl Zeiss) by two investigators (SM and FRB). Signals were counted for 200 tumour cells, as reported in the literature [19, 20, 37]. ALT-positive cases were identified when $\geq 5 \%$ of tumour cells exhibited large, very bright intranuclear foci of Tel-FISH signals. Endothelial cell nuclei were used for normal internal control purposes.

Selected images were converted into TIFF files and exported for telomere-specific image analysis using the TFL-Telo V2 software (BC Cancer Research Centre, Vancouver) [44]. As previously reported [37], we used TFL-Telo to ascertain the 
number of telomeres, the fluorescence signal intensities and the distribution of telomeres in neoplastic cells for each case. The mean intensity of telomeres, assumed to be proportional to their total length, was measured for each sample on $\geq 100$ neoplastic nuclei [37]. Other types of cell (e.g. infiltrating lymphocytes, endothelial cells) were excluded from the digital image analysis based on their morphological features.

The telomere fluorescence threshold (mean intensity $\approx$ 1000) was established by using positive and negative controls (previously analysed brain tumours with ALT activation and non-neoplastic brain tissues or telomerase/ALT negative gliomas, respectively). For comparison, we analysed 1 pilocytic astrocytoma (AP; WHO grade I), 1 primitive neuroectodermal tumour (PNET), 1 gliosarcoma (GSM, grade IV) and 1 oligoastrocytoma (OA; WHO grade II) (see Supplementary Table 1). Different telomeric profiles were identified in our cases (as shown Fig. 3). TFL-Telo analysis revealed significant differences in telomere length $(p<0.0001)$ between the positive and negative controls.

\section{Statistical analysis}

All data were recorded using Microsoft Excel 2016 and analysed using MedCalc statistical software (MedCalc Software Ltd, Belgium). A multifactor analysis of variance (ANOVA) was used to compare two or more groups. A $p$ value $<0.05$ was considered statistically significant. The correlation between genetic changes and patients' age was tested using linear regression and the $t$ test assuming unequal variances.

\section{Results}

Patients' demographic and clinical features are summarised in Table 1. We analysed 52 cases of pHGG (17 females and 35 males; age range 0.3 to 24 years, median 12.1 years). Based on the patients' neuropathological features, the sample included 32 glioblastomas (GBM, WHO grade IV), 10 K27-mutant diffuse midline gliomas (DMG, WHO grade IV), 5 highgrade astrocytomas (AA, WHO grade III) and 5 high-grade glioneuronal tumours (GNT). As previously stated, all cases included in this study were negative for IDH1-R132H. As expected [43], none of the patients over 12 years old showed any IDH1/2 gene point mutations apart from a biomolecular resemblance to malignant gliomas arising in $>14$-year olds and adults. All recruited cases had normal 1p/1q and 19q/19p, thereby excluding oligodendrogliomas.

We analysed the gene alterations involved in maintaining telomere length, such as TERTp, H3F3A and ATRX. We examined telomere length using a robust Tel-FISH assay to identify the activation of ALT. We measured telomere length on each sample using image analysis software, and correlated the presence of each specific mutation with the telomere length profile.

The detailed list of 52 samples, the tests performed on each sample and the results are provided in Table 1 in the Supplementary material.

\section{Genetic alterations involved in telomere maintenance mechanisms}

H3F3A mutations were found in $21 / 52$ cases (40\%). There were 10/52 (19\%) K27M mutations and 11/52 (21\%) G34R mutations. None of the samples analysed showed G34V mutation or other $H 3 F 3 A$ variants $(0 \%)$. ATRX nuclear loss was apparent in 16/52 (31\%) cases. Loss of ATRX expression was associated with the G34R mutation in $7 / 16$ cases, but in none of the K27M-mutated or TERTp-mutated samples. The results are summarised in Table 2. Genetic and molecular features, histological and clinical characteristics, and telomere length results are shown in Fig. 1.
Table 1 Summary of demographic and clinicopathological information of pHGG series

\begin{tabular}{lll}
\hline & Features & Frequency $(n=52 \mathrm{pHGG})$ \\
\hline \multirow{2}{*}{ Sex } & Male & $67.3 \%(35 / 52)$ \\
Age at diagnosis & Female & $32.7 \%(17 / 52)$ \\
& $\leq 5$ years & $17.3 \%(9 / 52)$ \\
& $6-10$ years & $25 \%(13 / 52)$ \\
& $10-15$ years & $17.3 \%(9 / 52)$ \\
Location & $15-24$ years & $40.4 \%(21 / 52)$ \\
& Mean = 12.1 years & \\
Histology & Supratentorial & $84.6 \%(44 / 52)$ \\
& Sub-/infratentorial & $15.4 \%(8 / 52)$ \\
& Glioblastoma & $61.6 \%(32 / 52)$ \\
& Diffuse midline glioma & $19.2 \%(10 / 52)$ \\
& Anaplastic astrocytoma & $9.6 \%(5 / 52)$ \\
& High-grade glioneuronal tumour & $9.6 \%(5 / 52)$
\end{tabular}


Table 2 Summary of results for ATRX, H3G34R, H3K27M and TERTp mutations, TERTp methylation and telomere elongation. The incidence of any alteration is reported for the whole cohort of pHGG and stratified by histology

\begin{tabular}{lllllll}
\hline Tumour type & ATRX nuclear loss & H3G34R-mut & H3K27M-mut & TERTp-mut & TERTp-meth & Telomere elongation \\
\hline pHGG $(n=52)$ & $30.7 \%(16 / 52)$ & $21.1 \%(11 / 52)$ & $19.2 \%(10 / 52)$ & $5.4 \%(2 / 37)$ & $21 \%(8 / 38)$ & $46.1 \%(24 / 52)$ \\
GBM $(n=32)$ & $46.8 \%(15 / 32)$ & $31.2 \%(10 / 32)$ & $0 \%$ & $3.1 \%(1 / 32)$ & $21.7 \%(5 / 23)$ & $56.2 \%(18 / 32)$ \\
DMG $(n=10)$ & $0 \%$ & $0 \%$ & $100 \%(10 / 10)$ & $0 \%$ & $37.5 \%(3 / 8)$ & $40 \%(4 / 10)$ \\
High-grade A $(n=5)$ & $20 \%(1 / 5)$ & $20 \%(1 / 5)$ & $0 \%$ & $20 \%(1 / 5)$ & $0 \%$ & $40 \%(2 / 5)$ \\
High-grade GNT $(n=5)$ & $0 \%$ & $0 \%$ & $0 \%$ & $0 \%$ & $0 \%$ & $0 \%$ \\
\hline
\end{tabular}

TERTp mutations were rare. The $\mathrm{C} 250 \mathrm{~T}$ mutation was found in $2 / 37$ cases $(5.4 \%)$, while hyper-methylation of TERTp on UTSS was detected in $8 / 38$ cases (21\%). Among these 8 UTSS hyper-methylated cases, 2 were H3.3/TERT/ ATRX-wildtype, 1 was associated with ATRX nuclear loss, $3 / 8$ with the K27M mutation and 2 with the G34R mutation. Representative results regarding the H3F3A and TERTp mutations, ATRX nuclear expression and UTSS methylation status on TERTp are shown in Fig. 2 in the Supplementary material.

G34R and K27M mutations were mutually exclusive and were never associated with TERTp mutations, as previously reported $[35,48,55]$. H3.3-G34R-mutant tumours were all supratentorial and associated with ATRX nuclear loss in $7 / 11$ cases $(64 \%)$. On the other hand, cases with the $\mathrm{K} 27 \mathrm{M}$ mutation (midline) were never associated with loss of ATRX nuclear expression. Eventually, patients with G34R-mutant pHGG had a significantly higher median age at diagnosis $(p=0.0014)$ than those with the K27M mutant (18.6 vs. 10.8 years) (Fig. 3 in the Supplementary materials), a finding consistent with previous reports $[37,55]$. The frequency of cases with alterations varied by histopathological variant (Table 2).

ATRX nuclear loss was more common in GBM (47\%). H3.3-G34R variant was detected mainly in GBM (31\%) and less frequently in high-grade astrocytomas (20\%), while H3.3$\mathrm{K} 27 \mathrm{M}$ variant was found exclusively in DMG (WHO).
TERTp hyper-methylation was only seen in $22 \%$ of GBM and $37 \%$ of DMG.

\section{ALT is frequently activated in pHGG}

pHGG were analysed using Tel-FISH to assess telomere length (Fig. 2). Telomere elongation due to the ALT mechanism was seen in $24 / 52$ cases $(46 \%)$, with a significant increase in the intensity of telomere fluorescence. In our cohort, ALT activation was associated exclusively with H3F3A or ATRX alterations. Three different ALT activation profiles were identified: $\mathrm{pHGG}$ with single tumour cells featuring large and very bright intranuclear telomere signals ( $\geq 5 \%$ of cells); $\mathrm{pHGG}$ with homogeneous areas of telomere elongation characterised by bright signals in large neoplastic regions; and pHGG with small, homogeneous telomere signals indicative of a basal-level telomere length. It is worth noting that the 2 cases carrying TERTp mutations were not associated with any increase in telomere length (Fig. 2), whereas 6/8 TERTp hyper-methylated pHGG showed telomere elongation via ALT, promoted by H3F3A mutations or ATRX loss. ALT was detected in GBM (56.2\%), DMG (40\%) and high-grade astrocytomas $(40 \%)$. There was no increase in telomere length in GNT or pilocytic astrocytomas, PNET, gliosarcomas or oligoastrocytomas used for control purposes.

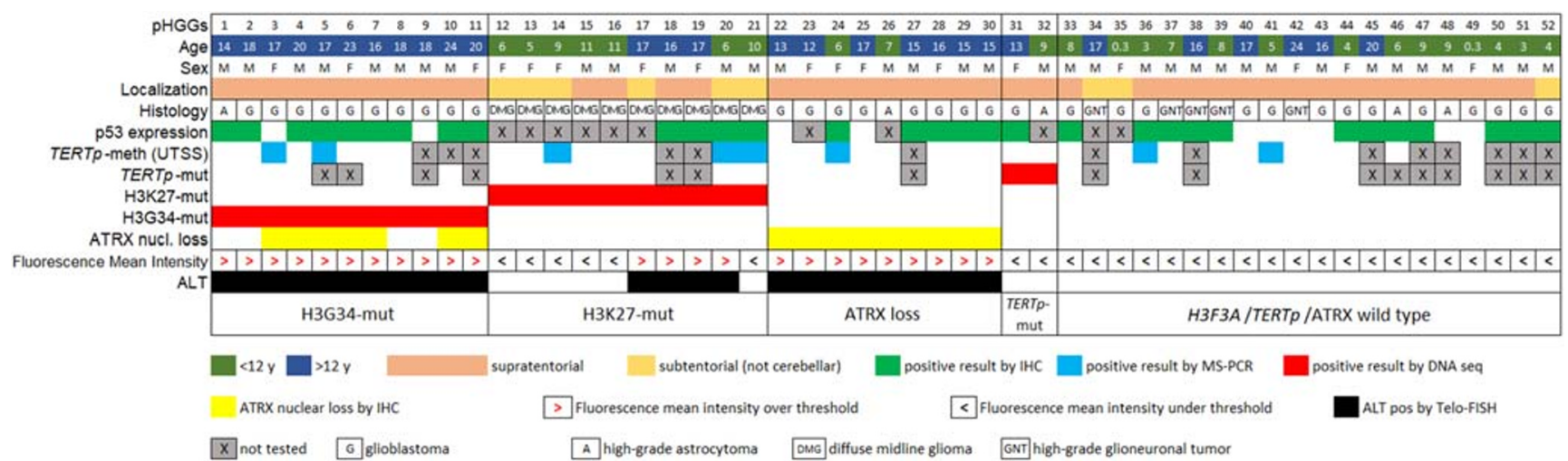

Fig. 1 Relevant clinico-pathological, genetic and molecular features of pHGG in our cohort, showing the increased telomere fluorescence intensity and ALT activation in different molecular subgroups of cases

with H3G34R mutations, H3K27M mutations, ATRX nuclear loss with H3.3-wt, TERTp mutations or no alterations on these genes 
Fig. 2 Panel of representative cases analysed for telomere elongation using Tel-FISH (magnification $\times 100)$. Green signals represent telomeres. a pHGG with large, very bright intranuclear foci of telomere signals in single cells. b Cases with telomere elongation featuring very bright signals in large neoplastic regions. $\mathbf{c}$ Cases with small and homogeneous telomere foci, indicative of

basal-level telomere length and no ALT

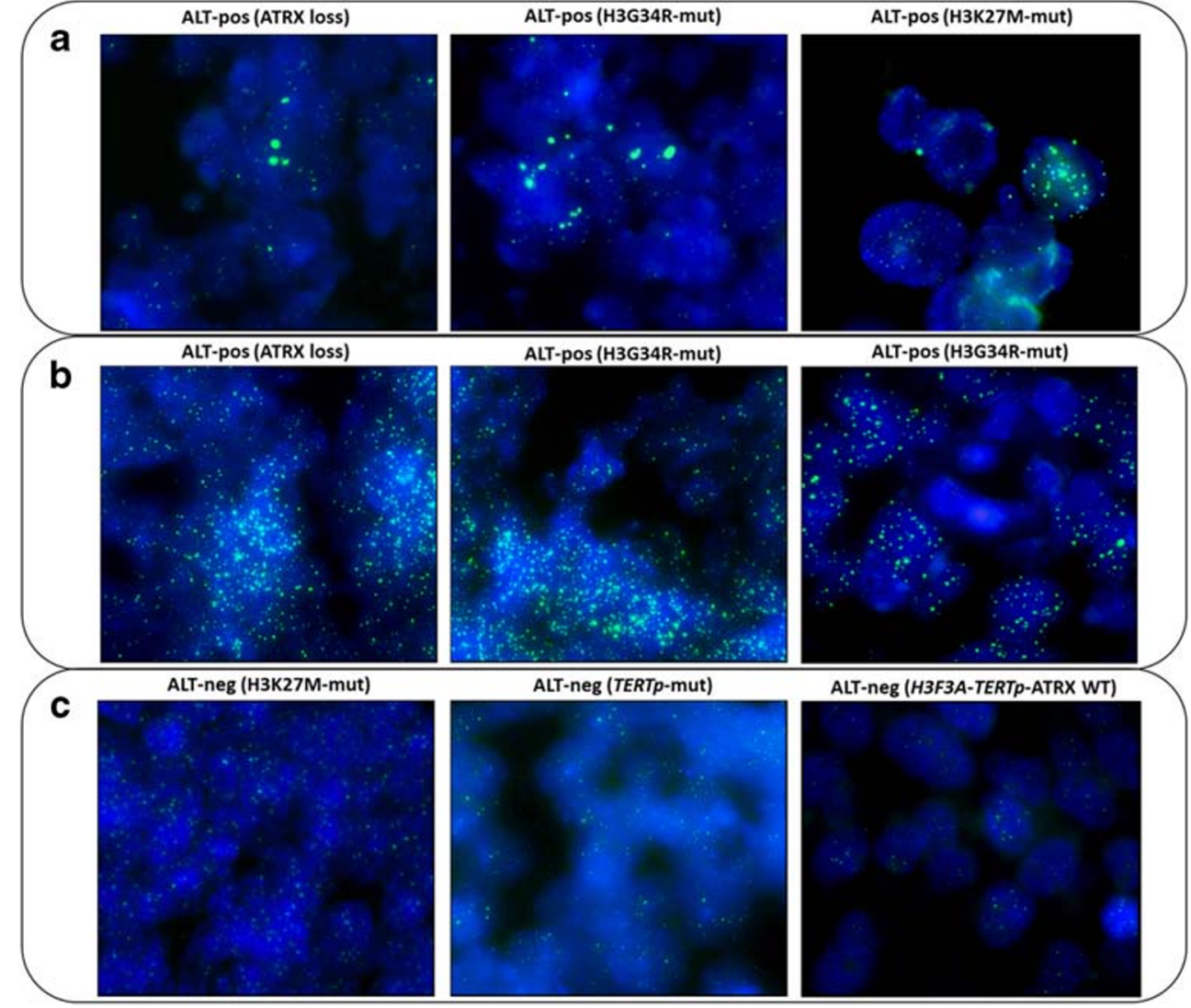

\section{H3.3 G34R and K27M mutations show different activation of ALT}

We analysed the relationships between telomere length and ATRX, H3G34, H3K27 and TERTp alterations (Fig. 3a), measuring telomere length with Tel-FISH and using TFL-Telo image analysis software, as described previously [37].

Telomere elongation was found in cases with $H 3 F 3 A$ mutations. Interestingly, all (11/11) G34R-mut pHGG showed

\section{a}

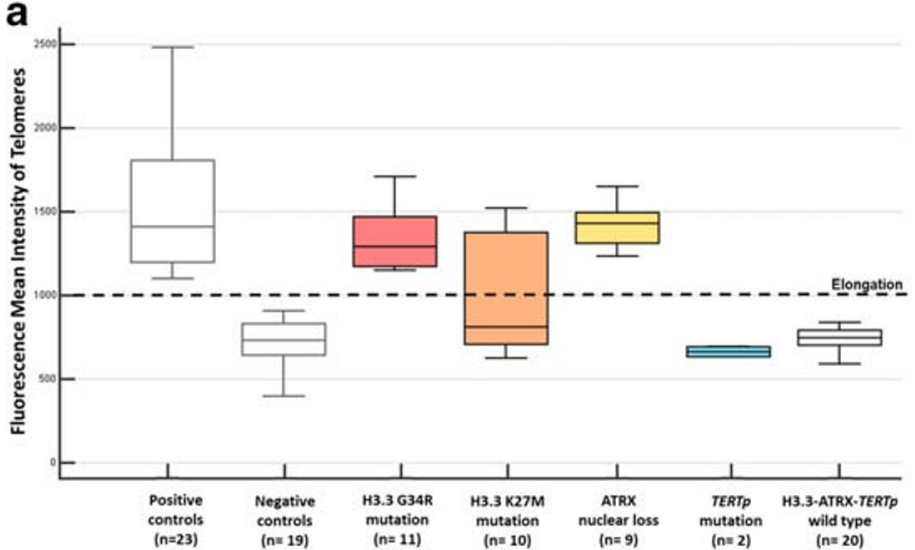

increased telomere length (Fig. 3b). Cases with H3.3-G34R variant showed ALT activation even in the absence of ATRX nuclear loss. Conversely, only 4/10 cases with the K27M mutation $(40 \%)$ had elongated telomeres, while the other $6 / 10$, $60 \%$ ) showed a telomere length comparable with that of negative controls. These results suggest that lysine 27 mutation is not sufficient to trigger ALT (Fig. 3a-b). Notably, all H3.3K27M-mutated pHGG showed a retained ATRX protein expression, as if ALT occurred irrespective of ATRX status. b

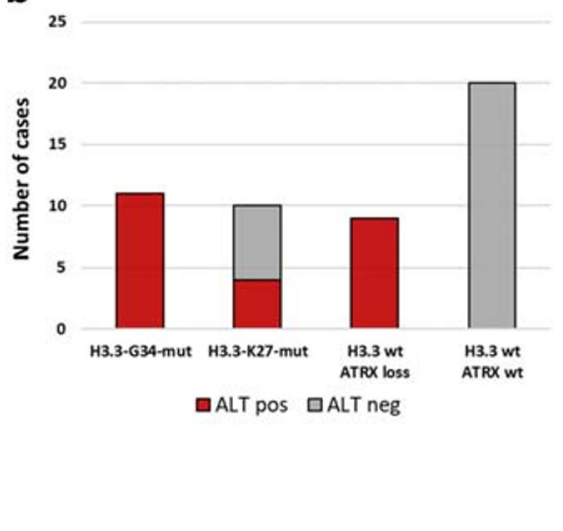

Fig. 3 Telomere length assessed in 52 pHGG. a Telomere fluorescence intensity quantified in different subgroups with H3.3-G34R mutations (red), H3.3-K27M mutations (orange), ATRX nuclear loss (yellow), TERT promoter mutations (light blue) or no alterations on these genes; positive and negative controls (grey boxes) were included to establish a threshold (dotted black line). b Number of cases with H3.3-G34-mut, H3.3-K27-mut, H3.3-wt with ATRX loss and H3.3/ATRX-wt, stratified according to ALT activation 
Telomere elongation was also found in all 9/9 cases with ATRX nuclear loss (100\%) (Fig. 3), always correlating with ALT activation irrespective of $H 3 F 3 A$ status. Telomere length was within control range in $\mathrm{pHGG}$ associated with TERTp mutations, possibly as a consequence of the lack of concurrent hyper-methylation of the TERT promoter on the UTSS region. Cases with no ATRX-H3F3A-TERTp alterations exhibited a normal telomere length (Fig. 3a).

Differences in the mean intensity of telomere fluorescence between the G34R- and K27M-mutant molecular subgroups were statistically significant $(p<0.05)$.

\section{Discussion and conclusion}

Maintenance of telomere length is a fundamental process by which cancer cells escape replicative senescence. Two different pathways sustain this process: reactivation of telomerase via TERT promoter mutations or hyper-methylation; and the ALT mechanism triggered by alterations on ATRX or $H 3 F 3 A$ $[1,2,5,14,19,20,26,36,38,46]$. In adult $H G G$, inactivating mutations in ATRX and TERTp are mutually exclusive, indicating that $A T R X$ loss contributes to telomere maintenance via the ALT phenotype. As to the association between $H 3 F 3 A$ mutations and ALT, a previous study showed that H3.3-G34R/V mutations were associated with global DNA hypo-methylation at the end of chromosomes. Neoplasms with G34R/V mutations have a significant overlap with those involving ATRX mutations and there is evidence of tumours with the H3.3-K27M mutation using ALT.

Our results show that $\mathrm{pHGG}$ frequently acquire a telomere maintenance mechanism via the ALT pathway (46\%), while telomerase reactivation via TERTp mutations/hypermethylation is not associated with telomere elongation. This finding demonstrates that ALT is the main telomere elongation pathway in $\mathrm{pHGG}$, unlike the case of adult HGG, which frequently shows telomerase reactivation triggered by TERTp mutations $(30-50 \%)[5,11,30]$. The literature shows that TERTp mutations are extremely rare in paediatric tumours $(<$ $5 \%)[2,11,23,26,30,35]$.

Previous data showed that cancer cells with reactivation of telomerase preferentially target short telomeres [1, 21] compared with normal samples $[2,50]$. A previous study showed that TERTp mutations do not activate telomerase expression enough to counteract telomere shortening [7], and this gives rise to cells with critically short and unprotected telomeres. Our results highlight that telomeres are not elongated in pHGG harbouring TERTp mutations, supporting that such mutations do not lead to any notable telomere elongation detected by quantitative FISH. A previous study on DNA methylation of the TERT promoter showed that UTSS hypermethylation was associated with tumour progression and a poor prognosis $[4,30,37]$. The present findings suggest that UTSS hyper-methylation alone $(2 / 8)$ is not sufficient to activate telomere elongation, as well as a TERTp mutation. This would mean that the maintenance of telomere length through telomerase reactivation requires a gradual upregulation of telomerase promoted by multiple steps [7]. Since UTSS hyper-methylation is only associated with telomere elongation and ultra-bright intranuclear telomere foci when concomitant H3.3 or ATRX alterations are present, therefore, telomere elongation must be driven by ALT pathway activation in our cases.

The present findings show a strong association between H3.3 mutations and ALT. Thus, all our G34R-mutant pHGG showed the ALT phenotype, while the K27M-mutant cases exhibited a much lower incidence of ALT activation (40\%). Interestingly, the G34R-mutant subgroup always used ALT to maintain telomere length, with either a simultaneous ATRX nuclear loss $(63.6 \%)$ or a retained ATRX (36.4\%). This finding suggests that the acquisition of a H3.3-glycine34 mutation might per se trigger the ALT mechanism, regardless of ATRX status. On the other hand, only a subset of H3.3-K27M-mutant gliomas triggered ALT, while the majority of cases $(60 \%)$ showed telomere shortening.

There were no differences as to TERTp or ATRX status between the K27M-mutant pHGG that activated ALT and those that did not, suggesting that other concomitant mutations or factors might contribute to activating ALT in such cases. As expected, cases with ATRX nuclear loss always trigger ALT, regardless of $H 3 F 3 A$ status, confirming a strong association with ALT in pHGG $[2,9,25,46]$. Moreover, our results also demonstrate that $H 3 F 3 A$ /ATRX/TERTp-wildtype pHGG never activate mechanisms for telomere elongation (this was seen in none of our 20 cases).

Despite this study's limitations, including the small size of our cohort and the absence of outcome correlations, the present findings add to what we know about the differences in the molecular profiles between adult HGG and pHGG. Our results provide preliminary evidence of differences in the incidence of telomere elongation in different molecular subgroups of pHGG, which may be relevant to the development of different therapeutic approaches to different $\mathrm{pHGG}$ populations. Research is currently underway on therapeutic strategies aimed at targeting molecules involved in telomere maintenance mechanisms. Early trials have produced encouraging results in adult HGG subtypes lacking histone H3.3 mutations, such as telomerase inhibitors for TERTp-mutant gliomas and ATR inhibitors for ALT-positive neoplasms with ATRX nuclear loss [23, 29]. If such ALT-targeted treatments will enter novel therapeutic protocols for molecular subgroups of pHGG, the analysis of telomere length would be necessary to pinpoint appropriate patients.

Potential ALT-targeted drugs have recently been proposed to hit heterochromatin formation driving the onset of ALT; the ATRX/DAXX/H3.3 complex, inhibitors of recombination 
factors (ATR, RAD52, SETDB1, FANCM) histone deacetylase inhibitors, G-quadruplex stabilisers; APB formation inhibitors; and other chromatin remodelling strategies $[10,15-17,31,34,52,56]$.

Our study revealed differences in the histone-defined subgroups of pHGG in terms of their telomeric profiles and ALT activation, improving our understanding of the association between histone mutations and ALT, and pointing to the possibility of using new targeted treatments for a subset of these tumours.

Although further validation on a larger prospective cohort will be necessary, our findings might contribute to the future use of highly selective therapeutic strategies for a subset of ALT-positive H3.3-mutated paediatric high-grade gliomas.

Acknowledgements The authors are grateful to Prof. Felice Giangaspero for his essential support, expert advice and critical reading of the manuscript.

Funding Open access funding provided by Università degli Studi di Roma La Sapienza within the CRUI-CARE Agreement. This research was supported by BimboTu ONLUS and Il Fondo di Gio ONLUS; Simone Minasi was supported by Fondazione Italiana per la Lotta al Neuroblastoma ONLUS.

Data availability Data available on request due to privacy/ethical restrictions.

\section{Compliance with ethical standards}

Conflict of interest The authors have no conflicts of interest to disclose.

Open Access This article is licensed under a Creative Commons Attribution 4.0 International License, which permits use, sharing, adaptation, distribution and reproduction in any medium or format, as long as you give appropriate credit to the original author(s) and the source, provide a link to the Creative Commons licence, and indicate if changes were made. The images or other third party material in this article are included in the article's Creative Commons licence, unless indicated otherwise in a credit line to the material. If material is not included in the article's Creative Commons licence and your intended use is not permitted by statutory regulation or exceeds the permitted use, you will need to obtain permission directly from the copyright holder. To view a copy of this licence, visit http://creativecommons.org/licenses/by/4.0/.

\section{References}

1. Akincilar SC, Unal B, Tergaonkar V (2016) Reactivation of telomerase in cancer. Cell Mol Life Sci 73:1659-1670

2. Barthel FP, Wei W, Tang M, Martinez-Ledesma E, Hu X, Amin SB, Akdemir KC, Seth S, Song X, Wang Q et al (2017) Systematic analysis of telomere length and somatic alterations in 31 cancer types. Nat Genet 49:349-357

3. Brosnan-Cashman JA, Yuan M, Graham MK, Rizzo AJ (2018) ATRX loss induces multiple hallmarks of the alternative lengthening of telomeres (ALT) phenotype in human glioma cell lines in a cell line-specific manner. PLoS One 13(9):e0204159. https://doi. org/10.1371/journal.pone.0204159

4. Castelo-Branco P, Sanaa C, Mack S et al (2013) Methylation of the TERT promoter and risk stratification of childhood brain tumours: an integrative genomic and molecular study. Lancet Oncol 14:534 542. https://doi.org/10.1016/S1470-2045(13)70110-4

5. Ceccarelli M, Barthel FP, Malta TM et al (2016) Molecular profiling reveals biologically discrete subsets and pathways of progression in diffuse glioma. Cell 164:550-563. https://doi.org/10.1016/j. cell.2015.12.028.

6. Cesare AJ, Griffith JD (2004) Telomeric DNA in ALT cells is characterized by free telomeric circles and heterogeneous t-loops. Mol Cell Biol 24(22):9948-9957. https://doi.org/10.1128/MCB. 24.22.9948-9957.2004

7. Chiba K, Lorbeer FK, Shain AH et al (2017) Mutations in the promoter of the telomerase gene TERT contribute to tumorigenesis by a two-step mechanism. Science 357(6358):1416-1420. https:// doi.org/10.1126/science.aao0535

8. Cohen AL, Holmen SL, Colman H (2013) IDH1 and IDH2 mutations in gliomas. Curr Neurol Neurosci Rep 13(5):345. https://doi. org/10.1007/s11910-013-0345-4

9. Danussi C, Promita B, Prosanna T et al (2018) Atrx inactivation drives disease-defining phenotypes in glioma cells of origin through global epigenomic remodeling. Nat Commun 9:1057. https://doi.org/10.1038/s41467-018-03476-6

10. Dilley RL (2015) \& Greenberg, R.A (2015). ALTernative telomere maintenance and cancer. Trends Cancer 1(2):145-156. https://doi. org/10.1016/j.trecan.2015.07.007

11. Diplas A, He X, Brosnan-Cashman JA et al (2018) The genomic landscape of TERT promoter wildtype-IDH wildtype glioblastoma. Nat Commun 9(1):2087. https://doi.org/10.1038/s41467-01804448-6

12. Dorris K, Sobo M, Onar-Thomas A et al (2014) Prognostic significance of telomere maintenance mechanisms in pediatric high-grade gliomas. J Neuro-Oncol 117(1):67-76. https://doi.org/10.1007/ s11060-014-1374-9

13. Ebrahimi A, Skardelly M, Bonzheim I et al (2016) ATRX immunostaining predicts IDH and $\mathrm{H} 3 \mathrm{~F} 3 \mathrm{~A}$ status in gliomas. Acta Neuropathol Commun 4:60. https://doi.org/10.1186/s40478-0160331-6

14. Episkopou H, Draskovic I, Van Beneden A et al (2014) Alternative lengthening of telomeres is characterized by reduced compaction of telomeric chromatin. Nucleic Acids Res 42:4391-4405. https://doi. org/10.1093/nar/gku1 14

15. Fan H-C, Chen C-M, Chi C-S et al (2019) Targeting telomerase and ATRX/DAXX inducing tumor senescence and apoptosis in the malignant glioma. Int J Mol Sci 20(1):200. https://doi.org/10. 3390/ijms20010200

16. Flynn RL, Cox KE, Jeitany M, Wakimoto H et al (2015) Alternative lengthening of telomeres renders cancer cells hypersensitive to ATR inhibitors. Science. 33:221-235. https://doi.org/10. 1126/science. 1257216

17. Gauchier M, Kan S, Barral A, Sauzet S et al (2019) SETDB1dependent heterochromatin stimulates alternative lengthening of telomeres. Sci Adv 5:eaav3673. https://doi.org/10.1126/sciadv. aav3673

18. Gielen GH, Gessi M, Hammes J, Kramm CM, Waha A, Pietsch T (2013) H3F3A K27M mutation in pediatric CNS tumors: a marker for diffuse high-grade astrocytomas. Am J Clin Pathol 139:345349. https://doi.org/10.1309/AJCPABOHBC33FVMO

19. Heaphy CM, De Wilde RF, Jiao Y et al (2011) Altered telomeres in tumors with ATRX and DAXX mutations. Science 333(6041):425. https://doi.org/10.1126/science.1207313

20. Heaphy CM, Subhawong AP, Hong SM et al (2011) Prevalence of the alternative lengthening of telomeres telomere maintenance 
mechanism in human cancer subtypes. Am J Pathol 179:16081615. https://doi.org/10.1016/j.ajpath.2011.06.018

21. Hemann MT, Strong MA, Hao LY, Greider CW (2001) The shortest telomere, not average telomere length, is critical for cell viability and chromosome stability. Cell 107:67-77

22. Henson JD, Cao Y, Huschtscha LI, Chang AC, Au AY, Pickett HA et al (2009) DNA C-circles are specific and quantifiable markers of alternative-lengthening-of-telomeres activity. Nat Biotechnol 27: 1181-1185. https://doi.org/10.1038/nbt.1587

23. Huang DS, Wang Z, He XJ, Diplas BH et al (2015) Recurrent TERT promoter mutations identified in a large-scale study of multiple tumor types are associated with increased TERT expression and telomerase activation. Eur J Cancer 51(8):969-976. https://doi. org/10.1016/j.ejca.2015.03.010

24. Jones C, Baker SJ (2014) Unique genetic and epigenetic mechanisms driving paediatric diffuse high-grade glioma. Nat Rev Cancer 14:651-661

25. Killela PJ, Reitman ZJ, Jiao Y, Bettegowda C et al (2013) TERT promoter mutations occur frequently in gliomas and a subset of tumors derived from cells with low rates of self-renewal. Proc Natl Acad Sci U S A 110:6021-6026. https://doi.org/10.1073/ pnas. 1303607110

26. Koelsche C, Sahm F, Capper D, Reuss D et al (2013) Distribution of TERT promoter mutations in pediatric and adult tumors of the nervous system. Acta Neuropathol 126:907-914. https://doi.org/10. 1007/s00401-013-1195-5

27. Lai TP, Wright WE, Shay JW (2018) Comparison of telomere length measurement methods. Philos Trans R Soc Lond Ser B Biol Sci 373(1741). https://doi.org/10.1098/rstb.2016.0451

28. Lang M, Jegou T, Chung I, Richter K, Münch S, Udvarhelyi A et al (2010) Three-dimensional organization of promyelocytic leukemia nuclear bodies. J Cell Sci 123:392-400. https://doi.org/10.1242/jcs. 053496

29. Lee J, Solomon DA, Tihan T (2017) The role of histone modifications and telomere alterations in the pathogenesis of diffuse gliomas in adults and children. J Neuro-Oncol 132(1):1-11. https://doi.org/ 10.1007/s11060-016-2349-9

30. Lee DD, Leão R, Komosa M, Gallo M et al (2019) DNA hypermethylation within TERT promoter upregulates TERT expression in cancer. J Clin Invest 129(1):223-229. https://doi.org/10.1172/ JCI121303

31. Lewis PW, Elsaesser SJ, Noh KM, Stadler SC, Allis CD (2010) Daxx is an H3.3-specific histone chaperone and cooperates with ATRX in replication-independent chromatin assembly at telomeres. Proc Natl Acad Sci U S A 107(32):14075-14080. https://doi.org/ 10.1073/pnas. 1008850107

32. Louis DN, Ohgaki H, Wiestler OD et al (2007) The 2007 WHO classification of tumours of the central nervous system. Acta Neuropathol 114(2):97-109. https://doi.org/10.1007/s00401-0070243-4

33. Lovejoy CA, Li W, Reisenweber S, Thongthip S et al (2012) Loss of ATRX, genome instability, and an altered DNA damage response are hallmarks of the alternative lengthening of telomeres pathway. PLoS Genet 8(7):e1002772. https://doi.org/10.1371/ journal.pgen. 1002772

34. Lu R, O'rourke JJ, Sobinoff AP et al (2019) The FANCM-BLMTOP3A-RMI complex suppresses alternative lengthening of telomeres (ALT). Nat Commun 10:2252. https://doi.org/10.1038/ s41467-019-10180-6

35. Mackay A, Burford A, Carvalho D et al (2017) Integrated molecular meta-analysis of 1,000 pediatric high-grade and diffuse intrinsic pontine glioma. Cancer Cell 32(4):520-537.e5. https://doi.org/10. 1016/j.ccell.2017.08.017

36. Mangerel J, Price A, Castelo-Branco P et al (2014) Alternative lengthening of telomeres is enriched in, and impacts survival of
TP53 mutant pediatric malignant brain tumors. Acta Neuropathol 128:853-862. https://doi.org/10.1007/s00401-014-1348-1

37. Minasi S, Baldi C, Pietsch T et al (2019) Telomere elongation via alternative lengthening of telomeres (ALT) and telomerase activation in primary metastatic medulloblastoma of childhood. J Neuro-Oncol 142:435-444. https://doi.org/10. 1007/s11060-019-03127-w

38. Nabetani A, Ishikawa F (2011) Alternative lengthening of telomeres pathway: recombination-mediated telomere maintenance mechanism in human cells. J Biochem 149:5-14. https://doi.org/ $10.1093 / \mathrm{jb} / \mathrm{mvq} 119$

39. Nonoguchi N, Ohta T, Oh JE et al (2013) TERT promoter mutations in primary and secondary glioblastomas. Acta Neuropathol 126:931-937. https://doi.org/10.1007/s00401-013-1163-0

40. Noushmehr H, Weisenberger DJ, Diefes K et al (2010) Identification of a $\mathrm{CpG}$ island methylator phenotype that defines a distinct subgroup of glioma. Cancer Cell 17(5):510-522. https:// doi.org/10.1016/j.ccr.2010.03.017.

41. Oganasian L, Karlseder J (2009) Telomeric armor: the layers of end protection. J Cell Sci 122:4013-4025. https://doi.org/10.1242/jcs. 050567

42. Ostrom QT, Gittleman H, Fulop J et al (2015) CBTRUS statistical report: primary brain and central nervous system tumors diagnosed in the United States in 2008-2012. Neuro-Oncology 17(Suppl 4): iv1-iv62

43. Pollack IF, Hamilton RL, Sobol RW et al (2011) IDH1 mutations are common in malignant gliomas arising in adolescents: a report from the Children's Oncology Group. Childs Nerv Syst 27(1):8794. https://doi.org/10.1007/s00381-010-1264-1

44. Poon SS, Lansdorp PM (2001) Quantitative fluorescence in situ hybridization (Q-FISH). Curr Protoc Cell Biol. Chapter 18, Unit18 14. Wiley. https://doi.org/10.1002/0471143030.cb1804s12

45. Reifenberger G, Weber RG, Riehmer V et al (2014) Molecular characterization of long-term survivors of glioblastoma using genome- and transcriptome-wide profiling. Int $\mathbf{J}$ Cancer 135(8): 1822-1831. https://doi.org/10.1002/ijc.28836

46. Rodriguez FJ, Brosnan-Cashman JA, Allen SJ et al (2019) Alternative lengthening of telomeres, ATRX loss and H3-K27M mutations in histologically defined pilocytic astrocytoma with anaplasia. Brain Pathol 29(1):126-140. https://doi.org/10.1111/bpa. 12646

47. Rubtsova MP, Vasilkova DP, Malyavko AN, Naraikina YV, Zvereva MI, Dontsova OA (2012) Telomere lengthening and other functions of telomerase. Acta Nat 2:44-61

48. Schwartzentruber J, Korshunov A, Liu XY et al (2012) Driver mutations in histone $\mathrm{H} 3.3$ and chromatin remodelling genes in paediatric glioblastoma. Nature 482:226-231. https://doi.org/10.1038/ nature 10833

49. Sturm D, Witt H, Hovestadt V, Khuong-Quang DA et al (2012) Hotspot mutations in H3F3A and IDH1 define distinct epigenetic and biological subgroups of glioblastoma. Cancer Cell 22:425-437. https://doi.org/10.1016/j.ccr.2012.08.024.

50. Wang Z, Rice SV, Chang TC, Liu Y, Liu Q, Qin N, Putnam DK, Shelton K, Lanctot JQ, Wilson CL et al (2019) Molecular mechanism of telomere length dynamics and its prognostic value in pediatric cancers. J Natl Cancer Inst. https://doi.org/10.1093/jnci/djz210

51. (2016) WHO classification of tumours of the central nervous system revised, 4th edn. In: Louis DN, Ohgaki H, Wiestler OD, Cavenee WK (eds) IARC, Lyon

52. Xu M, Qin J, Wang L et al (2019) Nuclear receptors regulate alternative lengthening of telomeres through a novel noncanonical FANCD2 pathway. Sci Adv 5(10):eaax6366. https://doi.org/10. 1126/sciadv.aax6366

53. Yamamoto H, Iwasaki T, Yamada Y et al (2018) Diagnostic utility of histone H3.3 G34W, G34R, and G34V mutant-specific 
antibodies for giant cell tumors of bone. Hum Pathol 73:41-50. https://doi.org/10.1016/j.humpath.2017.11.020

54. Yeager TR, Neumann AA, Englezou A, Huschtscha LI, Noble JR, Reddel RR (1999) Telomerase-negative immortalized human cells contain a novel type of promyelocytic leukemia (PML) body. Cancer Res 59(17):4175-4179

55. Yoshimoto K, Hatae R, Sangatsuda Y et al (2017) Prevalence and clinicopathological features of H3.3 G34-mutant high-grade gliomas: a retrospective study of 411 consecutive glioma cases in a single institution. Brain Tumor Pathol 34:103-112. https://doi.org/ 10.1007/s10014-017-0287-7
56. Zhang JM, Yadav T, Ouyang J (2019) Alternative lengthening of telomeres through two distinct break-induced replication pathways. Cell Rep 26(4):955-968.e3. https://doi.org/10.1016/j.celrep.2018. 12.102

57. Zhao S, Lin Y, Xu W, Jiang W et al (2009) Glioma-derived mutations in IDH1 dominantly inhibit IDH1 catalytic activity and induce HIF-1 $\alpha$. Science 324(5924):261-265. https://doi.org/10.1126/ science. 1170944

Publisher's note Springer Nature remains neutral with regard to jurisdictional claims in published maps and institutional affiliations. 